\title{
Characteristic prediction of colorectal cancer using multi- modality and multi-biomarker imaging
}

\author{
Hui Wang ${ }^{1}$ and Jia-he Tian ${ }^{2,3 *}$ \\ ${ }^{1}$ Nuclear Medicine Department, Hainan Branch of the Chinese PLA General Hospital, Sanya 572000, China \\ ${ }^{2}$ Nuclear Medicine Department, The Chinese PLA General Hospital, Beijing 100853, China \\ ${ }^{3}$ National Key-node Department of Medical Imaging, The Chinese PLA General Hospital, Beijing 100853, China
}

\begin{abstract}
To meet the clinical demand emerging with the changing paradigm in both diagnosis and treatment, the medical imaging techniques also proceeds into precision stage, becomes the major tasks beyond screening, early detection, staging and therapy evaluation. As heterogeneity of malignant tumors have major impacts on both scientific and clinical interests in CRC, multiple features are needed to be uncovered in order to better stratify and select the subgroups in view of their response status to the treatment and other biological characters. Multi-modality imaging, like PET/CT and PET/MR, using multi-biomarker specifically binding to key-targets in tumor and in microenvironment, might be the best choice for the goal.
\end{abstract}

\section{Introduction}

Colorectal cancer (CRC) is one of the most common types of cancer worldwide, which ranked the second in the list of cancer related death in well-developed western countries. The mortality of CRC has barely changed over the last 20 years, in part due to the approximately $50 \%$ of CRC remains undetected until late stage $[1,2]$. In spite of much improved surgical, chemical, radiation and targeted therapies, locoregional relapse and/or distant metastases, or metachronous secondary tumors are notified at an incidence of $3 \%-24 \%, 25 \%$, and $1.5 \%-10 \%$ respectively within the first 5 years after curative therapy [3].

For majority of CRC, chromosomal instability (CIN), microsatellite instability (MSI), and CpG-island methylator phenotype (CIMP) involving in different genetic or epigenetic abnormalities, activate the Ras/mitogen-activated protein kinase (MAPK) pathway (RAS-RAFMAPK) and the phosphoinositide 3-kianse (PI3K) pathway (PI3Kphosphatase and tensin homologue [PTEN]-Akt) [4]. These pathways are involved in CRC cell proliferation, differentiation, apoptosis, and cell invasion. Therefore, prediction of these different characterization of $\mathrm{CRC}$ by medical imaging is very useful for clinically precision medicine.

"Biomarker" is a term used widely in drug development and is defined as "a characteristic that is objectively measured and evaluated as an indicator of normal biological processes, pathogenic processes, or pharmacologic responses to a therapeutic intervention" [5]. Imaging biomarkers refers to those image findings able to reflect whether a patient is likely to respond to a treatment, or to detect and evaluate the treatment response, in correlation to the molecular mechanism hidden from ordinary investigation. Imaging biomarkers may directly illustrate molecular events and cellular characteristics, for example, assessing changes in the number of specific receptors or synthetic pathways, extracellular matrix signaling, intercellular signaling pathways, and gene expression, etc. which are specifically altered in particular tumor; or they may indirectly measure "downstream" sequelae of treatment or changes in molecular or cellular processes, for example, assessing tumor metabolism, and biophysics, cell density or blood flow. Those features revealing biochemical tumor-host interactions, are more and less "molecular" in nature and can be investigated by most of imaging methods.

This paper tries to associate what we have learnt from the molecular biology and medical imaging technology in an attempt to characterize CRC and to summarize what we could do by means of molecular imaging in order to provide clinically useful information for precision medicine, especially when targeted treatment is concerned.

\section{Imaging through indirect "biomarkers"}

In view of the current modification in CRC management, manufacturers and researchers made great efforts in recent years to improve the diagnostic performance of existing imaging modalities and to innovate new modalities to fulfill the emerging demands [6]. For the same reason, radiologists tried alternative ways in image acquisition and data handling to satisfy our clinical colleagues. Indeed, it has been reported that certain image findings are associated with underlying gene-expression patterns [7]. But so far, most attempts using conventional modalities stay mainly in indirect manner, due to lack of specific imaging biomarkers.

\section{CT imaging}

$\mathrm{CT}$ is the most widely available imaging platform. Conventional CT reflects anatomical and morphological features inside the body. Contrast enhancement, especially with multi-phase acquisition, could

${ }^{\star}$ Correspondence to: Jia-he Tian, Nuclear Medicine Department, The Chinese PLA General Hospital, Beijing 100853, China, Tel: 86-010-66936429; Fax: 86010-68161853; E-mail: tianjh@vip.sina.com

Key words: colorectal cancer, multi-biomarker, multi-modality, imaging, characterization

Received: October 15, 2018; Accepted: October 22, 2018; Published: October 29, 2018 
add diagnostic power of multi-slice CT (CECT). Because CT acquires its images in a straightforward way, and its image quality is stable and reliable, CT is recommended widely for follow-up of CRC after treatment, and evidence showed the virtual CT colongraphy had almost similar sensitivity as endoscopy in screening early CRC [8].

Precontrast images are used as baseline measurement of colon cancers, providing information about the tissue density, presence of fat, necrosis, calcium, or hemorrhage [9-11]. While after contrast injection, arterial blood supply, visceral perfusion, and venous drainage of interested lesion or tissue could be well depicted. In CRC, like some other types of tumors, neovascularization was reported to arise early in the adenoma-carcinoma sequence [12]. Angiogenesis plays an essential role in the process of growth and metastasis [13]. Blood flow (BF) could be used as an indirect biomarker for angiogenesis. In a CECT study, rectal cancer with low BF tended to be accompanied by synchronous metastatic lymph nodes or distant metastases, with poor overall survival as the consequence [14]. That is, however, conflict to what people expected for a hyper-perfused tumor, but other authors hypothesized that reduced $\mathrm{BF}$ are related to increasing interstitial fluid pressure and hypoxia in tumor tissue [15].

The recent advances in dual-energy computed tomography (DECT) simultaneously acquires datasets at two different photon spectra in a single scan. The iodine in the contrast agent can be distinguished from other materials owing to its stronger photoelectric absorption at low tube voltages near K-edge of iodine [16]. Furthermore, the dualenergy data can be used to generate a color-coded iodine overlay (IO) image that shows the distribution of iodine within the volume of tissue examined, and a 'pseudo' images of the host tissue without contrast (VNE) could be created with software to deduct IO from the acquired images. In an early study, single-phase DECT using color-coded IO and VNE images provide high accuracy in T-staging of CRC and suggest that VNE images could potentially replace the real pre-contrast (TNE) images. Replace TNE reduced the patient exposure to CT radiation by $57 \%$ [17]. Yang L et.al. study showed the radiomics signature was significantly associated with KRAS/NRAS/BRAF mutations. Clinical background, tumour staging, and histological differentiation were not associated with KRAS/NRAS/BRAF mutations in both cohorts [18].

\section{MR imaging}

Similar to modern CT, Magnetic resonance imaging (MRI) provides simultaneous assessment of morphological and functional features of a tumor. MRI is an extraordinary modality with great potential in multiple parametric (i.e. intrinsic bio-imaging biomarkers) imaging. With more than 300 acquisition sequences, each revealing different information based on chemical status of native proton, modern MRI is better than CT in revealing more valuable features of tumor, such as regional metabolism [19], circulation, cellular density [20], inter-lesion texture and 'bizarre' angiogenesis, interstitial $\mathrm{pH}[21], \mathrm{PO}_{2}$, mean vessel diameter [22] or intercellular distances in malignant tumor [23]. This capability of MRI is of great value since the interaction between tumor cells and neighbor cells in microenvironment surrounds it is known to impact tumor progression, even the distinct tumor cell subsets may contribute to the malignant phenotype [24]. All those makes multiparametric MRI a well-established most useful imaging modality, especially when the detection and characterization of soft tissue tumors is concerned [25]. Most of the aforementioned MRI findings could be more and less correlated with genetic and epigenetic counterparts [26].

One of the major shortcomings of contemporary MRI is its inherently relatively low sensitivity because only a small fraction of the nuclei in the sample actually contribute to measured signals [4]. To compensate this, 'smart contrast agents [27], and hyperpolarization technique were recently developed [28,29]. MRS is also a unique modality in its ability to provide chemically specific information on changes in molecular composition and structures.

Both ultrasound and nuclear medicine (to less extent) make use of indirect imaging biomarkers. While endoscopic ultrasound was proved useful in revealing transmural lesion and peri-intestinal tissue or lymph node involvement [30], which is not included in the current review. There is few acoustic contrast agent specific to known tumor biology except for blood flow [31].

\section{Radionuclide imaging}

Though most of radiolabeled tracers in nuclear medicine are more and less directed to molecular targets, ${ }^{[18]} \mathrm{F}$-fluorine or ${ }^{99} \mathrm{mTc}-\mathrm{MDP}$ for bone scan, and even ${ }^{[18]} \mathrm{F}-\mathrm{FDG}$ widely used for both clinical and basic science investigations, are in principle 'indirect' imaging biomarkers. In bony metastasis, the tracers accumulated in bone tissue around but not the metastatic lesion per se. The ${ }^{[18]}$ F-FDG takes advantage of "Warburg effect" [32], but it has long been recognized for its non-specific uptake pattern. Never the less, both ultrasound agent and nuclear medicine tracers have the advantage of carrying or directly delivering therapeutic effectors to the tumor $[33,34]$.

One technical might help to improve the diagnostic performance of indirect 'targeted' imaging. Texture analysis (TA) quantifies the spatial variations on the images [35-40], thus might add information concerning the heterogeneity within a tumor. It is a common finding with many studies that greater heterogeneity and higher fractal dimension was characteristic of malignant tumors, especially the more aggressive/advanced stage ones. Gneshaoh V, Gan et al found a coarse texture features may reflect the vasculature as defined by CD34 [41]. And coarse texture entropy on CT related to micrometastasis formation thus correlated with patient survival [42].

Texture analysis is proven to make ordinary imaging more informative, either CT or PET in detecting biological heterogeneity [43]. With texture analyzed, power in differential diagnosis between aggressive and non-aggressive/early stage tumors was proved with 83.3 $\%$ accuracy using CECT [44], so was the prediction of $65 \%$ increase risk of death by an increase of $6.5 \%$ via heterogeneity in ${ }^{[18]} \mathrm{F}-\mathrm{FDG}$ uptake [45]. Our texture analysis on 113 PET/CT cases derived from a previous multicenter trial on pulmonary lesions [46] found a $12 \%$ increase in diagnostic accuracy in comparison to randomized, blind, multi-readers' collective reading performance ( $83 \%$ vs $71 \%$ ). Using the same tracers, Wang et al firstly reported successful differentiation of a human CRC model with higher metastatic potential from another model from the same origin but low in metastases, and then proved the difference of the two tracers in evaluation of therapeutic effect [47]. Increased ${ }^{[18]}$ F-FDG SUV (max) and TW (PET/CT-based tumor width) (40\%) were associated in CRC tumors with TP53 and KRAS mutations, respectively [48].

\section{Imaging with direct biomarkers}

Traditionally, CRC is treated with surgery, chemotherapy and radiotherapy, depends on the type, location and stage of the tumor. However, due to heterogeneous nature of the tumor, CRC showed different biological behavior and way of evolution. As Esserman et al suggested, the term "cancer" should be reserved for those tumors of 
lethal progression if left untreated [49], it makes more sense to select the patients who are most likely to benefit from particular treatment and to deny those who not [50].

Currently, the selection of subgroup of patients is mainly depending on in vitro assessment of the related molecular biomarkers [51]. But the incomplete, fixed, frozen, out-of-living body samples might not be able to fully uncover the heterogeneity of molecular aberrations in the entire tumor, to say nothing about the acquired resistance during evolution of living cells. Multiple biopsies might solve the problem partially, as demonstrated in a report that F2108L mutation in MTOR causing resistant to everolimus was undetectable in the pretreatment tumor [52]. But molecular imaging, using those over expressed biomarker as imaging tracers, might serve the same purpose in a less invasive, more convenient, and direct way.

From clinical perspective, PET/CT (PET/MR) and MRI might be the modalities of choice when evaluating patients with a suspicion of recurrent disease, or of resistance to a certain regimen because of their best performance in revealing in vivo characteristic and confidence in prediction of disease status [53]. PET/CT uses radiotracers that directly bind to interested targets. Numerous radiotracers have been developed for a big variety of molecular and cellular targets, ranging from metabolism, proliferation, hypoxia, protein and lipid synthesis, membrane transporter of proteins, hormone receptors, tumor receptors, angiogenesis, apoptosis, and gene expression, etc.

\section{Proteins expression imaging}

Proteins are of enormous importance in living bodies for its being the main drivers of phenotypes. The aberrations on gene expression, mRNA and so on, could be expressed by relevant change of proteins. Quantification of proteins, their location, modifications, functions and their interactions would be a preferred target [54].

EGFR overexpression is present in approximately $80 \%$ of CRC cases, correlated with poor prognosis, shortened survival, and early recurrence $[55,56]$. A morpholino-[1241]-IPQA probe was reported able to bind the activated EGFR kinase adenosine triphosphate-binding site, but not to the inactive form [57]. Other tracers labeled by ${ }^{99 \mathrm{~m}} \mathrm{Tc}$ was able to differentiate sensitive and resistant mutation of EGFR [58]. The mechanism of acquired resistance to Everolimus was identified as a mutation in mTOR that prevented Everolimus from binding to mTOR. The mutant mTOR remained sensitive to direct mTOR kinase inhibition [59]. The latest report suggested a synthesis and labeling of an agent with potential of binding and imaging the pathway [60]. MRI has also been used to delineate constitutively activated EGFR in murine models, using an IgG antibody conjugated to iron oxide nanoparticles [61]. In another study, overexpression of LIM and $\mathrm{SH} 3$ domain protein 1 (LASP-1) in SW480 human CRC resulted in an aggressive phenotype and promoted cancer growth and metastasis [62]. Yang L showed multispectral imaging reveals hyper active TGF- $\beta$ signaling in colorectal cancer [63].

\section{miRNAs expression imaging}

MicroRNAs (miRNAs) may also be a promising target [64]. The first systematic analysis of miRNA expression in CRC was published in 2006 [65], followed by several studies confirming the dye-regulation of miRNAs. Chakrabarti et al designed, synthesized and evaluated ${ }^{99 \mathrm{~m}} \mathrm{Tc}$ and ${ }^{64} \mathrm{Cu}$ labeled probes specifically targeting oncogene mRNAs for SPECT or PET imaging [66].

One of the well-studied imaging markers is a small poly peptide: ArgGly-Asp (RGD) and its analogues. RGD, with favorable biodistribution properties and specific affinity to integrin receptor $\alpha v \beta 3$, plays an active role in angiogenesis, mediates cell attachment and many other cellular bioprocesses. Studies had demonstrated its usefulness in tumors and other situations when formation of new blood vessels is activated. ${ }^{99 \mathrm{~m}} \mathrm{Tc}$, ${ }^{68} \mathrm{Ga}$ and ${ }^{18} \mathrm{~F}$ labeled RGD were reported promising tracers for tumor imaging and monitoring anti-angiogenesis therapy, and are used widely for SPECT, PET and MRI imaging [67,68].

Low expression of epiregulin mRNA has been shown to be prognostic of improved OS for KRAS WT patients who did not receive EGFR-targeted therapy [69]. Tumor mRNA using a gene signature derived from liver metastases was proposed as a predictive biomarker for response to EGFR-targeted therapies [70]. A fraction of above cited studies demonstrated strong connection between abnormal imaging features, pinpointing particular abnormal molecular event in tumor, and their impact on the survival and clinical outcomes.

\section{Gene mutation imaging}

Single-nucleotide point mutations in codons 12 and 13 of exon 2 of KRAS gene were found in approximately $40 \%$ of patients with metastatic CRC [71]. Mutations in gene PI3KCA and loss of suppressor gene PTEN found in 15\%-20\% and 20\%-40\% of metastatic CRC were associated with resistance to cetuximab [72]. Those highly expressed mutations may be exploited as an imaging target for lesion detection.

According to the concept of tumor cell heterogeneity, highly metastatic cells are present as a sub-population in a primary tumor. In an in vivo selection of subpopulation of CRC cells of aggressive behavior, CD44 expression and c-MET activity represent greater tumorigenic potential, and an increased metastasis [73-75].

McKinley ET used preclinical models of CRC to demonstrate ${ }^{[18]}$ F-FLT PET as a sensitive predictor of response to (V600E) BRAF inhibitors. Because ${ }^{[18]}$ F-FLT PET predicted reduced proliferation associated with attenuation of BRAF downstream effectors, yet ${ }^{[18]}$ F-FDG PET did not, these data suggest that ${ }^{[18]}$ F-FLT PET may represent an alternative to ${ }^{[18]} \mathrm{F}-\mathrm{FDG}$ PET for quantifying clinical responses to BRAF inhibitors [76]. Schulte ML study showed $4^{\left[{ }^{[18 F}\right]}$ F-GLN PET selectively reflected pharmacodynamic response to BRAF inhibition when compared with 2-deoxy- $2^{[18 \mathrm{~F}]}$ fluoro-D-glucose PET, which was decreased non-specifically for all treated cohorts, regardless of downstream pathway inhibition [77].

Mutated KRAS caused higher ${ }^{[18]}$ F-FDG accumulation possibly by upregulation of GLUT1; moreover, HIF-1 $\alpha$ additively increased ${ }^{[18]}$ F-FDG accumulation in hypoxic lesions. ${ }^{[18]}$ F-FDG PET might be useful for predicting the KRAS status noninvasively [78].

\section{Conclusion}

Targeted therapies have improved the survival of patients with advanced CRC. However, further improvements in patient outcomes may be gained by the development of predictive biomarkers in order to select individuals who are most likely to benefit from treatment, thus personalizing treatment. Biomarkers can be prognostic, predictive target, or both. A predictive biomarker indicates the likelihood of response or resistance to a particular therapy, and a prognostic biomarker provides information on the outcome.

Ultimately, a comprehensive knowledge of mechanisms behind acquired resistance, coupled with the ability to diagnose the relevant aberrant molecular pathway in vivo, may lead to proper use and development of therapeutic strategies. Even most of techniques used with indirect imaging markers, the potential in scientific as well as 
clinical study will certainly be enhanced along with the improvements in acquisition and analysis technology, or it may serve as a component in the integrated CRC management with multi-tracer imaging and combination of targeted therapy.

\section{Acknowledgments}

The authors would like to express their sincere thanks to Drs. Yong Fang, Zhen Chang, Pen Yu, and Qiushi Ren for their assistance and suggestions in the reviewing. This work is partially supported by Projects on National Key-node Department of Medical Imaging, and several grants from Ministry of Science \& Technology (2011YQ030114, 2011CB707500), Hainan Key Foundation (ZDYF2016121), and PLA supporting research found (2014FC-TSYS-1004).

\section{Conflicts of interest}

The authors declare no conflict of interest in the article.

\section{References}

1. Bos JL, Fearon ER, Hamilton SR, Verlaan-de Vries M, van Boom JH, et al. (1987) Prevalence of ras gene mutations in human colorectal cancers. Nature 327: 293-297. [Crossref]

2. Siegel R, Naishadham D, Jemal A (2013) Cancer statistics, 2013. CA Cancer J Clin 63: 11-30. [Crossref]

3. Kekelidze M, D’Errico L, Pansini M, Tyndall A, Hohmann J (2013) Colorectal cancer: Current imaging methods and future perspectives for the diagnosis, staging and therapeutic response evaluation. World J Gastroenterol 19: 8502-8514.

4. Yarden Y, Sliwkowski MX (2001) Untangling the ErbB signalling network. Nat Rev Mol Cell Biol 2: 127-137. [Crossref]

5. Atkinson AJ Jr, Colburn WA, DeGruttola VG, DeMets DL, Downing GJ, et al. (2001) Biomarkers Definitions Working Group. Biomarkers and surrogate endpoints: Preferred definitions and conceptual framework. Clin Pharmacol Ther 69: 89-95.

6. Maas M, Rutten IJ, Nelemans PJ, Lambregts DM, Cappendijk VC, et al. (2011) What is the most accurate whole-body imaging modality for assessment of local and distant recurrent disease in colorectal cancer? A meta-analysis: imaging for recurrent colorectal cancer. Eur J Nucl Med Mol Imaging 38: 1560-1571.

7. Aerts HJ, Velazquez ER, Leijenaar RT, Parmar C, Grossmann P (2014) Decoding tumour phenotype by noninvasive imaging using a quantitative radiomics approach. Nat Commun 5: 4006-4010.

8. Hayano K, Fujishiro T, Sahani DV, Satoh A, Aoyagi T, et al. (2014) Computed tomography perfusion imaging as a potential imaging biomarker of colorectal cancer. World J Gastroenterol 20: 17345-1751.

9. Graser A, Johnson TR, Hecht EM, Becker CR, Leidecker C, et al. (2009) Dual-energy $\mathrm{CT}$ in patients suspected of having renal masses: Can virtual nonenhanced images replace true nonenhanced images? Radiology 252: 433-440.

10. Chae EJ, Song JW, Krauss B, Song KS, Lee CW, et al. (2010) Dual-energy computed tomography characterization of solitary pulmonary nodules. $J$ Thorac Imaging 25: 301310.

11. Sommer CM, Stampfl U, Bellemann N, Ramsauer S, Loenard BM, et al. (2010) Patients with lifethreatening arterial renal hemorrhage: CT angiography and catheter angiography with subsequent superselective embolization. Cardiovasc Intervent Radiol 33: 498-508.

12. Rak J, Mitsuhashi Y, Bayko L, Filmus J, Shirasawa S, et al. (1995) Mutant ras oncogenes upregulate VEGF/VPF expression: Implications for induction and inhibition of tumor angiogenesis. Cancer Res 55: 4575-4580.

13. Des Guetz G, Uzzan B, Nicolas P, Cucherat M, Morere JF, et al. (2006) Microvessel density and VEGF expression are prognostic factors in colorectal cancer. Meta-analysis of the literature. Br J Cancer 94:1823-1832.

14. Hayano K, Shuto K, Koda K, Yanagawa N, Okazumi S, et al. (2009) Quantitative measurement of blood flow using perfusion $\mathrm{CT}$ for assessing clinicopathologic features and prognosis in patients with rectal cancer. Dis Colon Rectum 2: 1624-1629.

15. Goh V, Halligan S, Wellsted DM, Bartram CI (2009) Can perfusion CT assessment of primary colorectal adenocarcinoma blood flow at staging predict for subsequent metastatic disease? A pilot study. Eur Radiol 19: 79-89.
16. Johnson TR, Krauss B, Sedlmair M, Grasruck M, Bruder H, et al. (2007) Material differentiation by dual energy CT: Initial experience. Eur Radiol 17: 1510-1517. [Crossref]

17. Chen CY, Hsu JS, Jaw TS, Wu DC, Shih MC, et al. (2014) Utility of the iodine overlay technique and virtual nonenhanced images for the preoperative $\mathrm{T}$ staging of colorectal cancer by dual-energy CT with tin filter technology. PLoS One 9: e113589.

18. Yang L, Dong D, Fang M, Zhu Y, Zang Y, et al. (2018) Can CT-based radiomics signature predict KRAS/NRAS/BRAF mutations in colorectal cancer? Eur Radiol 28 2058-2067.

19. Behar KL, Petroff OA, Prichard JW, Alger JR, Shulman RG (1986) Detection of metabolites in rabbit brain by 13C NMR spectroscopy following administration of [113C] glucose. Magn Reson Med 3: 911-920.

20. Lyng H, Haraldseth O, Rofstad EK (2000) Measurement of cell density and necrotic fraction in human melanoma xenografts by diffusion weighted magnetic resonance imaging. Magn Reson Med 43:828-823.

21. Gallagher FA, Kettunen MI, Day SE, Hu DE, Ardenkjaer Larsen JH, et al. (2008) Magnetic resonance imaging of $\mathrm{pH}$ in vivo using hyperpolarized $13 \mathrm{C}$-labelled bicarbonate. Nature 453: 940-943.

22. Troprès I, Grimault S, Vaeth A, Grillon E, Julien C, et al. (2001) Vessel size imaging. Magn Reson Med 45: 397-408. [Crossref]

23. Quarles CC, Gochberg DF, Gore JC, Yankeelov TE (2009) A theoretical framework to model DSC-MRI data acquired in the presence of contrast agent extravasation. Phys Med Biol 54: 5749-5766. [Crossref]

24. Cleary AS, Leonard TL, Gestl SA, Gunther EJ (2014) Tumour cell heterogeneity maintained by cooperating subclones in Wnt-driven mammary cancers. Nature 508 113-117.

25. Gore JC, Manning HC, Quarles CC, Waddell KW, Yankeelov TE (2011) Magnetic resonance in the era of molecular imaging of cancer. Magn Reson Imaging 29: 587600. [Crossref]

26. Thakur ML (2009) Genomic biomarkers for molecular imaging: predicting the future. Semin Nucl Med 39: 236-246. [Crossref]

27. Louie AY, Hüber MM, Ahrens ET, Rothbächer U, Moats R, et al. (2000) In vivo visualization of gene expression using magnetic resonance imaging. Nat Biotechnol 18: $321-325$.

28. Middleton H, Black RD, Saam B, Cates GD, Cofer GP, et al. (1995) MR imaging with hyperpolarized 3He gas. Magn Reson Med 33: 271-275. [Crossref]

29. Cudalbu C, Comment A, Kurdzesau F, van Heeswijk RB, Uffmann K, et al. (2010) Feasibility of in vivo $15 \mathrm{~N}$ MRS detection of hyperpolarized $15 \mathrm{~N}$ labeled choline in rats. Phys Chem Chem Phys 12: 5818-5823. [Crossref]

30. Liu D, Jin J, Tian J, Yang K (2015) Quality assessment and factor analysis of systematic reviews and meta-analyses of endoscopic ultrasound diagnosis. PLoS One 10: e0120911.

31. Dayton PA, Gessner RC, Phillips L, Shelton SE, Heath Martin K, et al. (2014) The implementation of acoustic angiography for microvascular and angiogenesis imaging. Conf Proc IEEE Eng Med Biol Soc 2014: 4283-4285. [Crossref]

32. WARBURG O (1956) On the origin of cancer cells. Science 123: 309-314. [Crossref]

33. Schoffelen R, Boerman OC, Goldenberg DM, Sharkey RM, van Herpen CM, et al (2013) Development of an imaging-guided CEA-pretargeted radionuclide treatment of advanced colorectal cancer: First clinical results. Br J Cancer 109: 934-942.

34. Capirci C, Rubello D, Chierichetti F, Crepaldi G, Carpi A, et al. (2004) Restaging after neoadjuvant chemoradiotherapy for rectal adenocarcinoma: Role of F18-FDG PET. Biomed Pharmacother 58: 451-457.

35. Ng F, Kozarski R, Ganeshan B, Goh V (2013) Assessment of tumor heterogeneity by CT texture analysis: can the largest cross-sectional area be used as an alternative to whole tumor analysis? Eur J Radiol 82: 342-348.

36. Cook GJ, Yip C, Siddique M, Goh V, Chicklore S, et al. (2013) Are pretreatment 18 F-FDG PET tumor textural features in non-small cell lung cancer associated with response and survival after chemoradiotherapy? J Nucl Med 54: 19-26.

37. Dong X, Xing L, Wu P, Fu Z, Wan H, et al. (2013) Three-dimensional positron emission tomography image texture analysis of esophageal squamous cell carcinoma: Relationship between tumor 18F-fluorodeoxyglucose uptake heterogeneity, maximum standardized uptake value, and tumor stage. Nucl Med Commun 34: 40-46. 
38. Ganeshan B, Goh V, Mandeville HC, Ng QS, Hoskin PJ, et al. (2013) Non-small cell lung cancer: histopathologic correlates for texture parameters at CT. Radiology 266: 326-336.

39. Ganeshan B, Miles KA, Young RC, Chatwin CR (2007) In search of biologic correlates for liver texture on portal-phase CT. Acad Radiol 14: 1058-1068. [Crossref]

40. Ganeshan B, Burnand K, Young R, Chatwin C, Miles K (2011) Dynamic contrastenhanced texture analysis of the liver: initial assessment in colorectal cancer. Invest Radiol 46: 160-168.

41. Goh V, Ganeshan B, Nathan P, Juttla JK, Vinayan A, et al. (2011) Assessment of response to tyrosine kinase inhibitors in metastatic renal cell cancer: $\mathrm{CT}$ texture as a predictive biomarker. Radiology 261: 165-171.

42. Tixier F, Le Rest CC, Hatt M, Albarghach N, Pradier O, et al. (2011) Intratumor heterogeneity characterized by textural features on baseline 18F-FDG PET images predicts response to concomitant radiochemotherapy in esophageal cancer. $J \mathrm{Nucl} \mathrm{Med}$ 52: 369-378.

43. Yu H, Caldwell C, Mah K, Mozeg D (2009) Coregistered FDG PET/CT-based textura characterization of head and neck cancer for radiation treatment planning. IEEE Trans Med Imaging 28: 374-383.

44. Harlow CA, Eisenbeis SA (1973) The analysis of radiographic images. IEEE Trans Comput 22: 678-689.

45. Mayerhoefer ME, Schima W, Trattnig S, Pinker K, Berger-Kulemann V, et al. (2010) Texture-based classification of focal liver lesions on MRI at 3.0 Tesla: a feasibility study in cysts and hemangiomas. J Magn Reson Imaging 32: 352-359.

46. Tian J, Yang X, Yu L, Chen P, Xin J, et al. (2008) A multicenter clinical trial on the diagnostic value of dual-tracer PET/CT in pulmonary lesions using 3'-deoxy-3'-18Ffluorothymidine and 18F-FDG. J Nucl Med 49:186-194.

47. Wang Hui, Zhang Jinming, Tian Jiahe, Qu Baolin, Li Tianran, et al. (2009) Using DualTracer PET to Predict the Biologic Behavior of Human Colorectal Cancer. J Nucl Med 50: $1857-1864$.

48. Chen SW, Lin CY, Ho CM, Chang YS, Yang SF, et al. (2015) Genetic Alterations in Colorectal Cancer Have Different Patterns on 18F-FDG PET/CT. Clin Nucl Med 40: 621-626. [Crossref]

49. Zhu Z, Miao W, Li Q, Dai H, Ma Q, et al. (2012) 99mTc-3PRGD2 for integrin receptor imaging of lung cancer: a multicenter study. J Nucl Med 53: 716-722. [Crossref]

50. Bos JL, Fearon ER, Hamilton SR, Verlaan-de Vries M, van Boom JH, et al. (1987) Prevalence of ras gene mutations in human colorectal cancers. Nature 327: 293-297. [Crossref]

51. Yoon YS, Kim JC (2014) Recent applications of chemosensitivity tests for colorectal cancer treatment. World J Gastroenterol 20: 16398-16408.

52. De Roock W, Claes B, Bernasconi D, De Schutter J, Biesmans B, et al. (2010) Effects of KRAS, BRAF, NRAS, and PIK3CA mutations on the efficacy of cetuximab plus chemotherapy in chemotherapy-refractory metastatic colorectal cancer: a retrospective consortium analysis. Lancet Oncol 11: 753-762.

53. Hurw Choi EK, Yoo IeR, Park HL, Choi HS, Han EJ, et al. (2012) Value of Surveillance (18)F-FDG PET/CT in Colorectal Cancer: Comparison with conventional imaging studies. Nucl Med Mol Imaging 46: 189-195.

54. Zhao L, Wang H, Liu C, Liu Y, Wang X, et al. (2010) Promotion of colorectal cancer growth and metastasis by the LIM and SH3 domain protein 1. Gut 59: 1226-1235. [Crossref]

55. Saadatian Z, Masotti A, Nariman Saleh Fam Z, Alipoor B, Bastami M, et al. (2014) Single-nucleotide polymorphisms within micrornas sequences and their 3' UTR target sites may regulate gene expression in gastrointestinal tract cancers. Iran Red Crescent Med J 16: e16659.

56. Mehlen P, Tauszig-Delamasure S (2014) Dependence receptors and colorectal cancer. Gut 63:1821-1829

57. Pal A, Glekas A, Doubrovin M, Balatoni J, Namavari M, et al. (2006) Molecular imaging of EGFR kinase activity in tumors with 124I-labeled small molecular tracer and positron emission tomography. Mol Imaging Biol 8: 262-77.

58. Shi J, Jia B, Liu Z, Yang Z, Yu Z, et al. (2008) 99mTc-labeled bombesin(7-14)NH2 with favorable properties for SPECT imaging of colon cancer. Bioconjug Chem 19: 1170-1178. [Crossref]

59. Hadjipanayis CG, Machaidze R, Kaluzova M, Wang L, Schuette AJ, et al. (2010) EGFRvIII antibody-conjugated iron oxide nanoparticles for magnetic resonance imaging-guided convection-enhanced delivery and targeted therapy of glioblastoma. Cancer Res 70: 6303-6312.
60. Yokota J (2000) Tumor progression and metastasis. Carcinogenesis 21: 497-503. [Crossref]

61. Kievit FM, Stephen ZR, Veiseh O, Arami H, Wang T, et al. (2012) Targeting of primary breast cancers and metastases in a transgenic mouse model using rationally designed multifunctional SPIONs. ACS Nano 6: 2591-2601.

62. Fisher R, Pusztai L, Swanton C (2013) Cancer heterogeneity: Implications for targeted therapeutics. Br J Cancer 108: 479-485.

63. Giannakis M, Hodis E, Jasmine Mu X, Yamauchi M, Rosenbluh J, et al. (2014) RNF43 is frequently mutated in colorectal and endometrial cancers. Nat Genet 46: 1264-1266.

64. Calin GA, Ferracin M, Cimmino A, Di Leva G, Shimizu M, et al. (2005) A MicroRNA signature associated with prognosis and progression in chronic lymphocytic leukemia N Engl J Med 353: 1793-1801.

65. Cummins JM, He Y, Leary RJ, Pagliarini R, Diaz LA Jr, et al. (2006) The colorectal microRNAome. Proc Natl Acad Sci U S A 103: 3687-3692. [Crossref]

66. Chakrabarti A, Zhang K, Aruva MR (2007) Radiohybridization PET imaging of KRAS G12D mRNA expression in human pancreas cancer xenografts with [(64)Cu]DO3Apeptide nucleic acid-peptide nanoparticles. Cancer Biol Ther 6: 948-956.

67. Kim HL, Sachin K, Jeong HJ, Choi W, Lee HS, et al. (2015) F-18 Labeled RGD Probes Based on Bioorthogonal Strain-Promoted Click Reaction for PET Imaging. ACS Med Chem Lett 6: 402-407. [Crossref]

68. Chen Q, Wang H, Liu H, Wen S, Peng C, et al. (2015) Multifunctional dendrimerentrapped gold nanoparticles modified with RGD Peptide for targeted computed tomography/magnetic resonance dual-modal imaging of tumors. Anal Chem 87: 3949 3956.

69. Khambata Ford S, Garrett CR, Meropol NJ, Basik M, Harbison CT, et al. (2007) Expression of epiregulin and amphiregulin and K-ras mutation status predict disease control in metastatic colorectal cancer patients treated with cetuximab. J Clin Oncol 25: 3230-3237.

70. Alvarez Chaver P, Otero-Estévez O, Páez de la Cadena M, Rodríguez-Berrocal FJ, Martínez-Zorzano VS (2014) Proteomics for discovery of candidate colorectal cancer biomarkers. World J Gastroenterol 20: 3804-3824.

71. Hurwitz H, Fehrenbacher L, Novotny W, Cartwright T, Hainsworth J, et all (2004) Bevacizumab plus irinotecan, fluorouracil, and leucovorin for metastatic colorectal cancer. N Engl J Med 350: 2335-2342.

72. Bianco R, Gelardi T, Damiano V, Ciardiello F, Tortora G (2007) Rational bases for the development of EGFR inhibitors for cancer treatment. Int J Biochem Cell Biol 39: 1416-1431. [Crossref]

73. Elliott VA, Rychahou P, Zaytseva YY, Evers BM (2014) Activation of c-Met and upregulation of CD44 expression are associated with the metastatic phenotype in the colorectal cancer liver metastasis model. PLoS One 9: e97432.

74. Li C, Wu JJ, Hynes M, Dosch J, Sarkar B, et al. (2011) c-Met is a marker of pancreatic cancer stem cells and therapeutic target. Gastroenterology 141: 2218-2227.e5.

75. Zaytseva YY, Rychahou PG, Gulhati P, Elliott VA, Mustain WC, et al. (2012) Inhibition of fatty acid synthase attenuates CD44-associated signaling and reduces metastasis in colorectal cancer. Cancer Res 72: 1504-1517.

76. Mansour H (2014) Cell-free nucleic acids as noninvasive biomarkers for colorectal cancer detection. Front Genet 5: 180-182.

77. Garouniatis A, Zizi-Sermpetzoglou A, Rizos S, Kostakis A, Nikiteas N, (2013) FAK $\mathrm{CD} 44 \mathrm{v} 6$, c-Met and EGFR in colorectal cancer parameters: tumour progression, metastasis, patient survival and receptor crosstalk. Int J Colorectal Dis 28: 9-18.

78. Baek S, Choi CM, Ahn SH, Lee JW, Gong G, et al. (2012) Exploratory clinical trial of (4S)-4-(3-[18F]fluoropropyl)-L-glutamate for imaging $\mathrm{xC}$ - transporter using positron emission tomography in patients with non-small cell lung or breast cancer. Clin Cancer Res 18: 5427-3547.

Copyright: (C2018 Wang H. This is an open-access article distributed under the terms of the Creative Commons Attribution License, which permits unrestricted use, distribution, and reproduction in any medium, provided the original author and source are credited. 